\title{
Enzyme-Responsive Release of Encapsulated Proteins from Biodegradable Hollow Capsules
}

\author{
Yuki Itoh, ${ }^{\dagger,+}$ Michiya Matsusaki, ${ }^{\dagger, \hbar}$ Toshiyuki Kida, ${ }^{\dagger, \S}$ and Mitsuru Akashi ${ }^{*, \dagger, \hbar, \S}$ \\ $\dagger$ Department of Applied Chemistry, Graduate School of Engineering, Osaka University, 2-1 \\ Yamada-oka, Suita 565-0871, Japan \\ $\$$ Center of Excellence (COE) Program for 21st Century, Osaka University, Japan
}

§Core Research for Evolutional Science and Technology (CREST), Japan Science and Technology Agency (JST), 4-1-8 Honcho, Kawaguchi-shi 332-0012, Japan

E-mail: akashi@chem.eng.osaka-u.ac.jp

Quantification of the reductive ends of Chitosan. Hollow capsules $(1.0 \mathrm{mg})$ were added to a chitosanase solution $\left(3.3 \mu \mathrm{M}, 10 \mathrm{~mL}, 4^{\circ} \mathrm{C}\right.$ or $\left.37^{\circ} \mathrm{C}\right)$. After $24 \mathrm{~h}$, the mixture was centrifuged. The Schales reagent was then incubated with Chitosan in the supernatant of the reaction systems for 15 min at $100{ }^{\circ} \mathrm{C}$. The enzymatic degradation was followed by a decrease in the absorption at $420 \mathrm{~nm}$. 


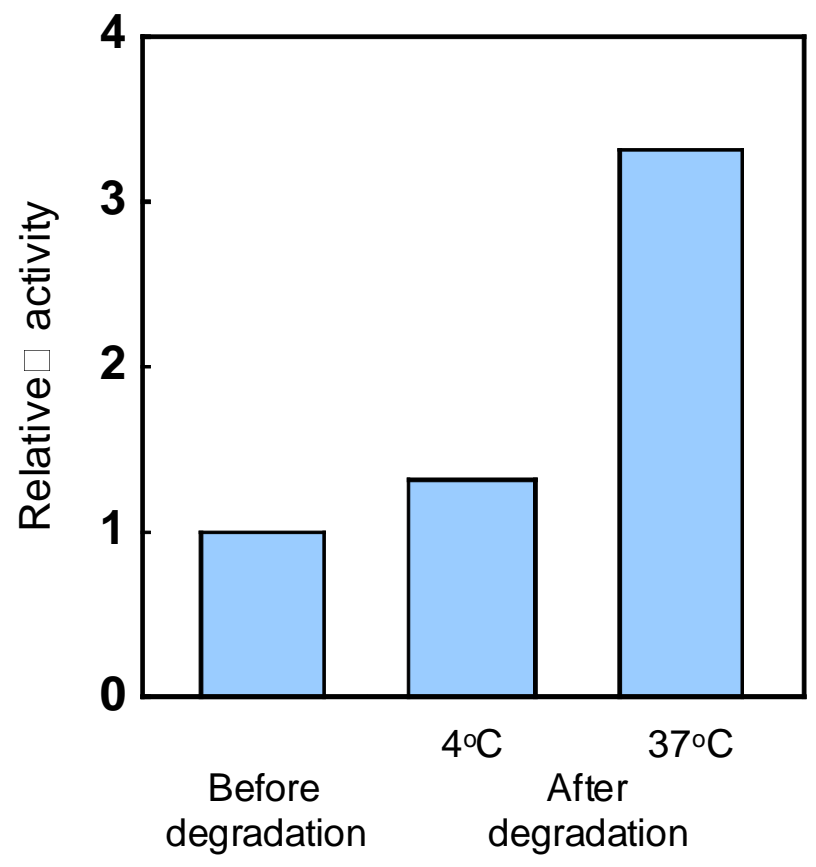

Figure S1. Quantification of the reductive ends in the polysaccharides by the Schales reagent before and after the enzymatic degradation of $(\mathrm{CT} / \mathrm{Dex})_{3}$ hollow capsules for $24 \mathrm{~h}$ at $4{ }^{\circ} \mathrm{C}$ or $37^{\circ} \mathrm{C}$.

The amount of reductive ends after the enzymatic reaction for $24 \mathrm{~h}$ at $4{ }^{\circ} \mathrm{C}$, a temperature at which the enzyme was inactive, was almost the same as that before the reaction (1.3 fold). However, the amount of reductive ends after the enzymatic reaction for $24 \mathrm{~h}$ at $37{ }^{\circ} \mathrm{C}$, a temperature at which chitosanase was active, was greater than that before the enzymatic degradation ( 3.3 fold). This result indicates that CT in hollow capsules was degraded by chitosanase. 